\title{
Hepatozelluläres Karzinom
}

\section{Hepatocellular Carcinoma}

\author{
Gesprächsleiter: J. Klempnauer, Hannover \\ Teilnehmer: C.E. Broelsch, Essen M. Galanski, Hannover G. Gerken, Essen
}

\section{Frage 1: Welche Pathomechanismen führen zur Ausbildung eines hepatozellulären Karzinoms?}

Broelsch: Chronische Hepatitis-Virus-B(HBV)- und Hepatitis-Virus-C(HCV)-Infektionen stellen das Hauptrisiko für die Entwicklung eines hepatozellulären Karzinoms (HCC) dar. Neben Alkohol sind weitere, noch nicht genauer bekannte Risikofaktoren chemische Karzinogene und hormonelle Faktoren. Da in einem HCC häufig eine Überexpression von «transforming growth factor» (TGF) alpha oder c-myc und cfos nachgewiesen wird, stellen vermutlich auch diese und andere genetischen Faktoren, wie Protein-X-Expression oder Adhäsionsmoleküle ICAM-1 und HLA-II-Moleküle, eine Komponente der Karzinogenese des HCC dar. Interessanterweise führt die Aktivierung von $\beta$-Catenin in Kooperation mit c-myc-ausgelösten Signalwegen zur Entwicklung von hochdifferenzierten HCCs mit einer niedrigen Rate an chromosomaler Instabilität.

Die meisten Hepatozyten befinden sich in einem ruhenden Stadium. Eine Induktion der Zellzyklusprogression von der G0/G1- in die S-Phase erfolgt durch verschiedene Faktoren wie Zytokine, Wachstumsfaktoren, Onkogenüberexpression oder Inaktivierung der Suppressorgene Rb-Protein und p53. Sie wird von der HBV/HCV-Infektion unterstützt. Daraus entwickeln sich DNA-Mutationen und chromosomale Rearrangements, die als Hauptdeterminante der Zelltransformation gelten. Entziehen sich solche partiell transformierten Hepatozyten der Apoptose und der immunologischen Kontrolle, entwickeln sich daraus vollständig transformierte Zellen, die dann mit Hilfe von weiteren genetischen Faktoren und insbesondere unter Einfluss von Angiogenese zu neoplastischen malignen Zellen mit hohem Metastasierungspotential heranwachsen.

Eine Inkorporation der HBV-DNA in die Leberzelle kann zu einem chromosomalen Rearrangement führen. So konnte eine Integration der HBV-DNA in die 17p13-chromosomale Region, in der das p53-Tumorsuppressorgen lokalisiert ist, oder in das Cyclin-A-Protein, welches eine wesentliche Rolle in der G1/S- und G2/M-Phase aufweist, gezeigt werden. Die Rolle der HCV-Infektion ist noch unklar. Vermutet wird ein direkter Einfluss auf die transkriptionale Regulation der cmyc-, c-fos-, Rb- und IFN- $\beta$-Gene, auf die transformierende Eigenschaften der NS3-Serinprotease und auf die Apoptosegene Fas und TNF-R. Besonders interessant scheint dabei der 1b-HCV-Genotyp zu sein.

Gerken: Weltweit gehört das HCC zu den häufigsten menschlichen Tumoren mit einer hohen Inzidenz, vor allem in Zentral- und Südafrika sowie in Südostasien. In den letzen Jahren wird auch in Nordamerika und Europa eine Zunahme der HCC-Inzidenz im Zusammenhang mit der Zunahme der HCV-bedingten Leberzirrhose beobachtet.

Die ätiologischen Faktoren sind neben der Infektion mit HBV und HCV auch Faktoren wie Alkohol, Aflatoxin B1, Nikotin sowie metabolische Störungen wie Hämochromatose, Alpha1-Antitrypsinmangel. Selten ist die Inzidenz des HCC bei autoimmuner Hepatitis, primär biliärer Zirrhose und Morbus Wilson zu beobachten.

Pathogenetisch ist für die HCC-Entstehung die Sequenz von chronischer Entzündung, gesteigerter Leberregeneration und nachfolgenden genetischen Veränderungen relevant. Als genetische Mechanismen gelten die Aktivierung von Onkogenen, die Inaktivierung von Tumorsuppressorgenen und chromosomale Rearrangements. Für HBV und bestimmte Umweltfaktoren wie Aflatoxin wird auch ein direktes onkogenes Potential postuliert. Ein deutlich gesteigertes Risiko für die Entwicklung eines HCC liegt insbesondere für die Kombination verschiedener ätiologischer Faktoren vor, wie z.B. Virus plus Alkohol, Virus plus Aflatoxin, Alkohol plus Rauchen. Zusammenfassend bleibt festzuhalten, dass das HCC sich in

\begin{tabular}{ll}
\hline KARGER & ( ) 2003 S. Karger GmbH, Freiburg \\
Fax +497614520714 & Accessible online at: \\
$\begin{array}{l}\text { E-mail Information@Karger.de } \\
\text { www.karger.com }\end{array}$ & www.karger.com/cga
\end{tabular}

Prof. Dr. med. Jürgen Klempnauer 
bis zu 90\% der Fälle auf dem Boden einer Leberzirrhose entwickelt, welche somit ätiologisch als eine Präkanzerose zu werten ist.

\section{Frage 2: Welche Anforderungen sind an die präoperative Diagnostik zu stellen?}

Broelsch: Für die HBV- und HCV-infizierte Risikopopulation gelten die perkutane Ultraschalluntersuchung (US) mit einer Sensitivität von $71 \%$ und einer Spezifität von $93 \%$ und die Alpha-Fetoprotein(AFP)-Bestimmung im Serum mit einer Sensitivität von 40-64\% und einer Spezifität von $75-91 \%$ als Basisuntersuchungen, die in 6-monatigen Abständen wiederholt werden sollen. In einem italienischen Kollektiv von 1125 Hepatitis-infizierten Patienten konnte unter Berücksichtigung dieser Empfehlung bei 5,9\% ein HCC, das sich jedoch nur in weniger als $50 \%$ in einem noch resektablen Stadium befand, nachgewiesen werden.

Wird eine suspekte Läsion $<1 \mathrm{~cm}$ entdeckt, sollte der US alle 3 Monate mit dem Ziel eines Nachweises der Tumorgrößenzunahme, die als Indikator für Malignität gilt, wiederholt werden. Bei suspekten Läsionen $>1 \mathrm{~cm}$ und/oder pathologischen AFP-Serumwerten muss weiterführende bildgebende Diagnostik erfolgen.

Die Methoden mit der größten Sensitivität und Spezifität sind helikale Computertomographie (CT) mit arterieller und portaler Phase und das dynamische Gadolinum-verstärkte Kernspintomogramm (magnetic resonance imaging; MRI) in Multiphasentechnik und Nachweis eines «tumor enhancement» während der arteriellen Phase. Bei Patienten mit einer Leberzirrhose, die für eine Lebertransplantation vorgesehen sind, sollte während der Wartezeit das MRI alle 6 Monate wiederholt werden, um ein HCC möglichst früh zu entdecken und es dann bis zur Organverfügbarkeit als Überbrückung lokal zu behandeln. Die diagnostische Bedeutung einer Feinnadelbiopsie ist umstritten. Während ein negatives Ergebnis ein HCC nicht ausschließt und mit einer Inzidenz von 2-3\% für Stichkanalmetastasen zu rechnen ist, können molekulare Charakteristika des Biopsates wertvolle therapeutische Selektionskriterien liefern.

Trotz hoch entwickelter diagnostischer Verfahren bleibt eine sichere Unterscheidung zwischen einem HCC und einem Regeneratknoten in einer Zirrhoseleber schwierig. Bei 20-60\% der $\mathrm{HCV} / \mathrm{HBV}$-assoziierten HCCs ist mit einem synchronen oder metachronen multizentrischen Tumorwachstum zu rechnen. Der Vergleich der Daten der bildgebenden Diagnostik, des intraoperativen Befundes und der histologischen Ergebnisse zeigt, dass ein signifikanter Anteil der HCCs präoperativ nicht adäquat eingestuft wird.

Gerken: Der entscheidende laborchemische Parameter für den Nachweis eines HCC ist ein deutlich ansteigender AFPSerumwert. Es ist jedoch anzumerken, dass insbesondere bei kleinen Tumoren in bis $\mathrm{zu} 30 \%$ aller neu diagnostizierten HCCs das AFP negativ ist und zum anderen moderate AFPAnstiege im Bereich zwischen 20 und $400 \mathrm{ng} / \mathrm{ml}$ auch bei Patienten mit Leberzirrhose ohne nachweisbares $\mathrm{HCC} z u$ finden sind.

Die Sensitivität des AFP liegt zwischen 50 und 70\%, die Spezifität bei $80-90 \%$. Erst bei AFP-Werten über $400 \mathrm{ng} / \mathrm{ml} \mathrm{kann}$ die Diagnose eines HCC als nahezu diagnostisch gesichert gelten, zumal diese Patienten dann meist eine größere Tumormasse, einen multilokulären oder diffusen Befall oder eine neu entstandene Pfortaderthrombose aufweisen.

Der AFP-Marker sollte aufgrund seiner eingeschränkten diagnostischen Wertigkeit als klinisch etablierter HCC-Tumormarker vornehmlich mit bildgebenden Verfahren diagnostisch kombiniert werden.

Als bildgebendes Verfahren sind die Abdomensonographie mit und ohne Kontrastmittel sowie die verschiedenen radiologischen Techniken wie MRI und Angio-CT der Leber etabliert. Aktuell scheint aufgrund der Entwicklung neuer superparamagnetischer Eisenpartikel als leberspezifische Kontrastmittel das Leber-MRI die sensitivste Technik zum Nachweis von Lebertumoren sein.

Als Staging zum HCC-Screening bei Patienten mit fortgeschrittenen chronischen Lebererkrankungen empfiehlt sich in der klinischen Praxis die Bestimmung der Kombination aus AFP sowie Abdomensonographie in 3- bis 6-monatigen Abständen.

Eine histologische Sicherung von Lebertumoren sollte insbesondere in unklaren Fällen, bei niedrigem AFP oder bei nicht eindeutiger Bildgebung erfolgen, wobei die Gefahr von Stichkanalmetastasen $\mathrm{zu}$ berücksichtigen ist. Zur histologischen Diagnosesicherung ist die US- oder CT-gesteuerte Feinnadelpunktion technisch die Methode der Wahl.

Galanski: Die präoperative Bildgebung beinhaltet drei Aufgaben: 1) den Tumornachweis, 2) die Lokalisations- und Ausbreitungsdiagnostik einschließlich einer Beurteilung der Leberhilusstrukturen, der Portal- und Lebervenen in Hinblick auf die Resektabilität und 3) den Ausschluss einer extrahepatischen Tumormanifestation.

Der Tumornachweis und die intrahepatische Ausbreitungsdiagnostik beim HCC sind wegen des variablen Erscheinungsbilds und der meist zugleich vorhandenen Zirrhose anspruchsvolle Aufgaben. Die Schwierigkeit der Lokalisationsdiagnostik in der zirrhotischen Leber ist durch eine hohe Prävalenz von Regeneratknoten, eine hohe Dysplasierate der Regeneratknoten und eine hohe Prävalenz okkulter Tumoren (jeweils bis zu 30\%) gekennzeichnet. Das HCC zeigt in allen Modalitäten (US, CT, MRI) ein breites Befundspektrum; es reicht von «typischen» hypervaskularisierten Läsionen mit oder ohne Pseudokapsel über multilokuläre Herde bis hin zu einer disseminierten Tumorinfiltration der Leber, die sich dem Nachweis nahezu entziehen kann. Hypervaskularisierte Tumoren sind in der Regel besser nachweisbar als hypovaskularisierte. 
Alle bildgebenden Verfahren - Sonographie, CT und MRI erreichen nur unbefriedigende Sensitivitätswerte. Die differierenden Angaben zur Sensitivität (60-90\%) sind in erster Linie auf das variable Erscheinungsbild und eine unterschiedliche Zusammensetzung der Patientenkollektive zurückzuführen. Bei allen Modalitäten basiert die Diagnostik zu einem wesentlichen Teil auf dem Nachweis der arteriellen Versorgung im Gegensatz zur überwiegend portalvenösen Versorgung des Parenchyms.

Die Sonographie (Farb-Doppler-US) ist in der Regel das primäre bildgebende Verfahren in der HCC-Diagnostik; sie ist allerdings stark untersucher- und geräteabhängig. Die am besten geeigneten Nachweisverfahren sind die biphasische Spiral-CT-Untersuchung und die MRI, gegebenenfalls mit spezifischem Kontrastmittel. Da die meisten HCC-Knoten hypervaskularisiert sind, liefert die arterielle Phase der biphasischen Spiral-CT die verlässlichsten Informationen. Die MRI ist der CT insofern überlegen, als sie nicht nur auf das Kriterium der arteriellen Versorgung angewiesen ist, sondern über das Signalverhalten und die Aufnahme RES-spezifischer Kontrastmittel die Tumordetektion verbessern und zur Abgrenzung gegenüber Regeneratknoten beitragen kann. Ein Nachteil der MRI ist allerdings die höhere Artefaktanfälligkeit.

Welchem komplementärem Verfahren zur Sonographie, der MRI oder CT, letztlich der Vorzug in der präoperativen Diagnostik gegeben wird, hängt nicht zuletzt von der verfügbaren Gerätetechnik, der persönlichen Erfahrung des Untersuchers und von Patientenfaktoren ab und muss oftmals im Einzelfall entschieden werden.

Eine Beteiligung der großen Pfortader- oder Lebervenenäste kann mit allen Verfahren nachgewiesen werden. Unsicher hingegen ist der Nachweis der peripheren Gefäßinvasion, dem durchaus prognostische Bedeutung zukommt.

Der Nachweis oder Ausschluss einer extrahepatischen Tumormanifestation ist - abgesehen von der regionalen Lymphknotenmetastasierung - unproblematisch. Methode der Wahl ist hierfür die CT, die in einem Untersuchungsgang Auskunft über den pulmonalen und abdominellen Status einschließlich des Stammskeletts geben kann. Die Beurteilung der regionalen Lymphknoten ist nach wie vor ein ungelöstes Problem, da als Dignitätskriterium lediglich Form und Größe der Lymphknoten mit unbefriedigender Trennschärfe herangezogen werden können.

\section{Frage 3: Welche Kriterien müssen für eine chirurgische Intervention erfüllt sein (Indikation zur Resektion, Indikation zur Transplantation)?}

Broelsch: Mit dem Ziel der Identifikation von Prognosefaktoren und der Diskriminierung von verschiedenen therapeutischen Optionen wurden mehrere klinische «Scoring»-Systeme entwickelt. Von begrenzter Bedeutung sind das TNM(Tumor, Lymphknoten, Metastasen)-Stadium, der Karnofsky-Index oder die Child-Pugh-Klassifikation, da sie nur eine einzelne Variable berücksichtigen. Auch die sehr verbreitete OkudaKlassifikation (Bilirubin, Albumin, Aszites, Tumormasse) hat Schwächen, da sie nicht zwischen den einzelnen Tumorstadien unterscheiden kann. Die «Barcelona-Clinic Liver Cancer Staging»-Klassifikation sieht unter der Berücksichtigung von klinischen Parametern und verschiedenen Behandlungsmodalitäten vier Stadien vor. So werden für Patienten in frühen Stadien (asymptomatisches solitäres $\mathrm{HCC}<5 \mathrm{~cm}$ oder weniger als 3 HCC-Knoten mit einem Maximaldurchmesser von < $3 \mathrm{~cm}$, potentiell kurative Behandlungsmöglichkeiten wie Resektion, Lebertransplantation oder lokale Ablation) gute 5Jahres-Überlebensraten erwartet. Für Patienten, die diese Kriterien nicht erfüllen, werden 3-Jahres-Überlebensraten von weniger als $10 \%$ kalkuliert. Neue multidimensionale Systeme wie CLIP-Score oder Chevret-Klassifikation bedürfen weiterer klinischer Prüfung.

Ein generell akzeptabler therapeutischer Algorithmus, der weltweit Berücksichtigung finden könnte, existiert nicht. Da randomisierte, kontrollierte, klinische Studien fehlen, können allgemein geltende «First-line»-Therapieempfehlungen nicht gegeben werden. Vielmehr werden zentrumsspezifische Empfehlungen, basierend auf Ergebnissen einzelner Kohortenstudien, eigener Erfahrung und zur Verfügung stehenden Ressourcen gegeben.

Grundsätzlich gelten Leberresektion und Lebertransplantation als die einzigen Verfahren mit einer potentiell kurativen Option.

Eine onkologisch adäquate Resektion ist das Verfahren der ersten Wahl für nicht zirrhotische Patienten mit $\mathrm{HCCs}<5 \mathrm{~cm}$ Durchmesser. Patienten mit einer Child-Pugh-A-Leberzirrhose und nur mäßig erhöhten Bilirubinwerten $(<1,9 \mathrm{mg} / \mathrm{dl})$ ohne portale Hypertension gelten ebenfalls als potentielle Resektionskandidaten. Intraoperativ soll ein US mit dem Ziel der Entdeckung eines multizentrischen Tumorwachstums erfolgen. Auch in erfahrenen Zentren ist die Resektionsrate mit $16 \%$ (USA, Europa) bis 33\% (Asien, Taiwan) niedrig. Für Child-Pugh-B- und -C-Patienten mit einem Tumor $<5 \mathrm{~cm}$ oder 3 Knoten mit einem Maximaldurchmesser $<3 \mathrm{~cm}$ gilt in den meisten Zentren die Lebertransplantation als das Verfahren der Wahl.

Aus großen hepatobiliären Zentren werden 3- und 5-JahresÜberlebensraten von 68-76\% und 51-68\% mit einer Mortalität von $<2 \%$ berichtet. Als «minimal cutoff value» gilt eine 5Jahres-Überlebensrate von 50\%. Die Ergebnisse der Leberresektion werden signifikant durch den Grad der vorliegenden Leberzirrhose bestimmt. Auch Child-Pugh-A-Patienten haben ein hohes Risiko, nach der Resektion einen schwer therapierbaren Aszites zu entwickeln. Bei selektionierten Patienten mit unzureichender funktioneller Leberreserve kann durch eine Embolisation der rechten Pfortader eine sichere sekundäre Resektabilität erzielt werden.

Bei Vorliegen eines HCC $>5 \mathrm{~cm}$ ist die Datenlage kontrovers. Während einzelne Zentren über vergleichbare Erfahrungen wie 
bei Tumoren $<5 \mathrm{~cm}$ berichten, stellen andere deutlich schlechtere Daten vor. Nur einzelne Studien geben Erfahrungen mit HCCs $>10 \mathrm{~cm}$ wieder. Aus Japan wird bei diesem problematischen Kollektiv eine Mortalität von 14,3\% und eine 3-Jahresbzw. 5-Jahres-Überlebensrate von 25 bzw. 8,2\% berichtet.

Einheitlich ist die Erfahrung der 3-Jahres- bzw. 5-Jahres-Tumorrezidivrate von 50 bzw. 75-100\%. Das Rezidiv manifestiert sich in Form von intrahepatischen Metastasen, die als langsam wachsendes, zum Zeitpunkt der Erstoperation nicht erkanntes synchrones multizentrisches Karzinom gelten, oder in Form einer metachronen multilokulären Manifestation. Auch die Häufigkeit des HCC-Rezidivs wird vom Grad der begleitenden Leberzirrhose bestimmt, da sie als Präkanzerose gilt. Ein besonders hohes Rezidivrisiko haben HCV-positive Patienten. Der präventive Effekt von intraarteriellem Jod131-markierten Lipiodol oder IFN- $\alpha$ wird zur Zeit diskutiert. Theoretisch stellt die Lebertransplantation die optimale Therapieoption dar, da sie sowohl das HCC als auch die begleitende Leberzirrhose eliminiert. Die initialen Ergebnisse der Lebertransplantation, die mit einer sehr liberalen Indikationsstellung eingesetzt wurde, waren mit einer 60\% igen Tumorrezidivrate innerhalb der ersten 2 Jahre nach der Operation und einer 1-Jahres-Überlebensrate von $42-71 \%$ enttäuschend. Unter Berücksichtigung strengerer Selektionskriterien und bei Begrenzung der Indikation zur Lebertransplantation auf Patienten mit kleinen HCCs konnten im weiteren Verlauf bessere Ergebnisse, vergleichbar mit denen bei Zirrhotikern ohne HCCs, erzielt werden.

Bei Berücksichtigung der immanenten Organknappheit und der theoretischen Möglichkeit des frühen Tumorrezidivs unter der Immunsuppression wurde mit Hilfe von verschiedenen Kriterien versucht, die HCC-Patienten, die für eine Lebertransplantation in Frage kommen, zu identifizieren. In den meisten Transplantationszentren wird zur Zeit der MilanScore, der in die American Liver Tumor Study Group Modified TNM Staging Classification integriert ist, angewandt. Das Vorliegen eines einzelnen Tumors $<5 \mathrm{~cm}$ und das Vorliegen von weniger als 3 Tumoren mit einem Durchmesser $<3 \mathrm{~cm}$ gelten als Kriterien für eine Lebertransplantation. Unter Berücksichtigung dieser Kriterien lassen sich 4-Jahres-Gesamtüberlebensraten und 4-Jahres-Überlebensraten mit Tumorfreiheit von 85 und $92 \%$ erzielen. Die Behandlungsergebnisse der Patienten, die als Kandidaten für eine Lebertransplantation gelten, werden durch die Wartezeit auf ein Spenderorgan und die damit verbundene Möglichkeit einer Tumorprogression negativ beeinflusst. Die Lebendorganspende eröffnete hier neue Möglichkeiten, da sie einerseits die Wartezeit auf ein passendes Organ verkürzt und andererseits die Option für eine potentielle Indikationserweiterung bietet.

Gerken: Prinzipiell stellt die chirurgische Therapie der HCCs die einzige Möglichkeit einer kurativen Behandlung dar. Chirurgische Verfahren beim HCC umfassen vor allem die Leberteilresektionen, die Lebertransplantation und eventuell die
Kombination von Radiofrequenzablation und Resektionsverfahren.

Die therapeutischen Optionen bei Vorliegen eines HCC hängen von der Tumorzahl, der Tumorgröße, der Leberfunktion, der Gefäßbeteiligung sowie der Metastasierung ab.

Eine Indikation zur Resektion ist nur gegeben bei Child-AZirrhose ohne Komorbidität, wenn ein solitärer Herd, d.h. ein unilateraler Befall, vorliegt.

Bei multiplen Herden (maximal 3 mit einer Größe $<3 \mathrm{~cm}$ ) oder bei einem solitären Herd (Größe $<5 \mathrm{~cm}$ ) ist bei fehlender Gefäßinvasion und einer Leberfunktion Child B oder C die Transplantation zu präferieren. Hierbei können heute 3Jahres-Überlebensraten von bis zu $80 \%$ und 5-Jahres-Überlebensraten von bis zu $75 \%$ erreicht werden. Bei dem aktuellen Organmangel werden die Ergebnisse jedoch durch die lange Wartezeit verschlechtert. Die Leberlebendspende stellt hierfür eine neue, sorgfältig abzuwägende Therapieoption in entsprechenden Zentren dar. Generell kann die Überlegenheit der Transplantation gegenüber der Resektion auf die Beseitigung der Grunderkrankung und die niedrigere Rezidivgefahr zurückgeführt werden.

\section{Frage 4: Welche nichtoperativen Behandlungsstrategien kommen in Betracht?}

Broelsch: Das Spektrum umfasst viele lokale und systemische Therapieoptionen. Als lokale Maßnahmen gelten die Chemoembolisation, die Alkoholinjektion sowie die Radiofrequenz-, Laser- oder Ultraschallablation. In einzelnen Patienten lassen sich mit diesen Verfahren sehr gute Behandlungsergebnisse erzielen. Die Ergebnisse der systemischen Chemotherapie sind enttäuschend. Systemische Therapie mit Somatostatin oder gentherapeutische Ansätze z.B. mit antiAFP-gerichteten Adenoviren sind unter Erprobung.

Gerken: Bei Leberherden $>5 \mathrm{~cm}$ sowie multiplen $(>4)$ Herden und bei nachweisbarer Gefäßbeteiligung stehen zunächst als lokal ablative Verfahren die perkutane Alkoholinjektion, die transarterielle Chemoembolisation und die Radiofrequenz-Thermoablation zur Verfügung. Sollte es unter diesen Therapiemaßnahmen bei jungen Patienten und fehlender Progression der Grunderkrankung zu einem kompletten «downstaging» kommen, kann erneut eine Indikation zur Resektion oder zur Lebertransplantation erwogen werden. Bei extrahepatischer Metastasierung, Komorbidität, schlechtem Allgemeinzustand und fortgeschrittener Child-C-Leberzirrhose bleiben nur palliative Ansätze zu diskutieren. Jedoch bietet weder die klassische Chemotherapie noch die Gabe von Tamoxifen, Somatostatin, Pravastatin oder Interleukin einen entscheidende Überlebensvorteil. Über neue Strategien wie die Gabe von Antiangiogenese-Faktoren, Gentherapie und Immuntherapie liegen noch keine ausreichend gesicherten klinischen Daten vor. 
Galanski: Die einzigen kurativen Therapieoptionen beim HCC sind die Resektion und die Transplantation. Allerdings sind nur etwa $25 \%$ der HCCs resektabel. Nichtoperative Therapieverfahren haben in der Regel einen palliativen Ansatz.

Regionale Therapiekonzepte sind die perkutane Alkoholinjektion, die thermoablativen Verfahren wie die Radiofrequenzablation oder die laserinduzierte Thermotherapie und die transarterielle Chemoembolisation.

Mit lokal abladierenden Verfahren kann bei Tumoren $<3 \mathrm{~cm}$ in einem hohem Prozentsatz eine vollständige Tumornekrose erzielt werden. Die umfangreichsten und längsten Erfahrungen liegen mit der perkutanen Alkoholinjektion vor. Bei Tumoren $<5 \mathrm{~cm}$ werden Überlebensraten von über $90 \%$ nach 1 Jahr und von $70 \%$ nach 3 Jahren erzielt. Nach 5 Jahren ist allerdings in über zwei Drittel der Fälle mit einem Rezidiv zu rechnen, wobei es sich in der Regel um neue Herde im Sinne eines multifokalen Tumorwachstums handelt. Ähnliche Ergebnisse sind für die Radiofrequenzablation oder die laserinduzierte Thermotherapie zu erwarten.

Die transarterielle Chemoembolisation kommt in erster Linie für multilokuläre Tumoren Betracht, setzt aber eine ausreichende Leberleistung voraus. Bei korrekter Indikationsstellung können Überlebensraten von bis zu 75\% nach 1 Jahr und $30 \%$ nach 3 Jahren erzielt werden.

Ein fortgeschrittenes Tumorstadium (TNM-Stadium 3, Okuda 3) und eine fortgeschrittene Zirrhose (Child-Pugh C) gelten als Kontraindikationen für eine regionale Therapie gleich welcher Art. Diese Patienten kommen nur für eine symptomatisch supportive Therapie in Betracht.

Die systemische Chemotherapie hat sich als nicht wirksam erwiesen, auch nicht nach einer Resistenzmodulation der Monosubstanzen. Gleiches gilt für die Hormontherapie.

\section{Teilnehmer}

Prof. Dr. med. Dr. h.c. mult. Christoph E. Broelsch

Klinik für Allgemein- und Transplantationschirurgie Universitätsklinikum Essen

Hufelandstraße 55

D-45122 Essen

E-mail christoph.broelsch@uni-essen.de

Prof. Dr. med. Michael Galanski

Diagnostische Radiologie

Medizinische Hochschule Hannover

Carl-Neuberg-Straße 1

D-30625 Hannover

Tel. +49 51153234 21, Fax -38 85

E-mail galanski.m@mh-hannover.de

Prof. Dr. Guido Gerken

Abteilung für Gastroenterologie und Hepatologie

Zentrum für Innere Medizin, Universitätsklinikum Essen

Hufelandstraße 55

D-45122 Essen

Tel. +492017233610

E-mail g.gerken@uni-essen.de 